\title{
'Cleft sign' of severe lipohypertrophy
}

\author{
$\mathrm{CM} \mathrm{Ng}{ }^{*}$, OL Chui, SC Tiu
}

Hong Kong Med J 2015;21:475.e3-4

DOI: 10.12809/hkmj154528

A 45-year-old woman complained of increasing truncal obesity in October 2014. Physical examination revealed a 'buttock-like' configuration of her lower abdomen, with a midline 'cleft' flanked on either side by two rubbery pendulous masses (Fig 1).

She had diabetes mellitus and had been injecting herself with Protaphane HM 26 IU (Novo Nordisk A/S, Denmark) before bed, and Actrapid HM 15/20/20 IU (Novo Nordisk A/S, Denmark) 3 times a day, since 2007. Knowing that insulin injection was often accompanied by weight gain, she attributed these subcutaneous masses, which had enlarged slowly over the past 3 years, to abdominal obesity.

The physical finding was more compatible with severe lipohypertrophy secondary to repeated insulin injections. Although both conditions involve an accumulation of adipocytes in the subcutaneous layer, a 'cleft' is normally not seen in abdominal obesity, except in patients with a history of vertical sub-umbilical incision (Fig 2). In contrast, in severe insulin-injection lipohypertrophy, there is often this tell-tale sign of a 'cleft', because injections are made into either side of the abdomen with sparing of the midline area.

Lipohypertrophy is a common complication of insulin therapy and occurs in up to two thirds of patients using insulin. ${ }^{1}$ Once a small area of lipohypertrophy forms, patients tend to favour this area for injection since it is less painful although it results in growth of these masses. Risk factors include use of long needles with wide-bore diameter, re-use of the same needle, multiple injections, high doses of insulin, and repeated injections into the same sites. ${ }^{1}$ In our own clinic patient cohort, $89(71 \%)$ out of 125 patients using vial insulin and 36 (72\%) of 50 patients using an insulin pen have lipohypertrophy identifiable by palpation, albeit usually to a lesser degree. Ultrasound or magnetic resonance imaging examination may identify an even higher incidence.

Further injection into these areas is not advised because insulin absorption from these fat masses can be erratic; this results in hypoglycaemia or a need for higher doses of insulin. ${ }^{2}$ Regular examination of the injection sites for this local complication should be

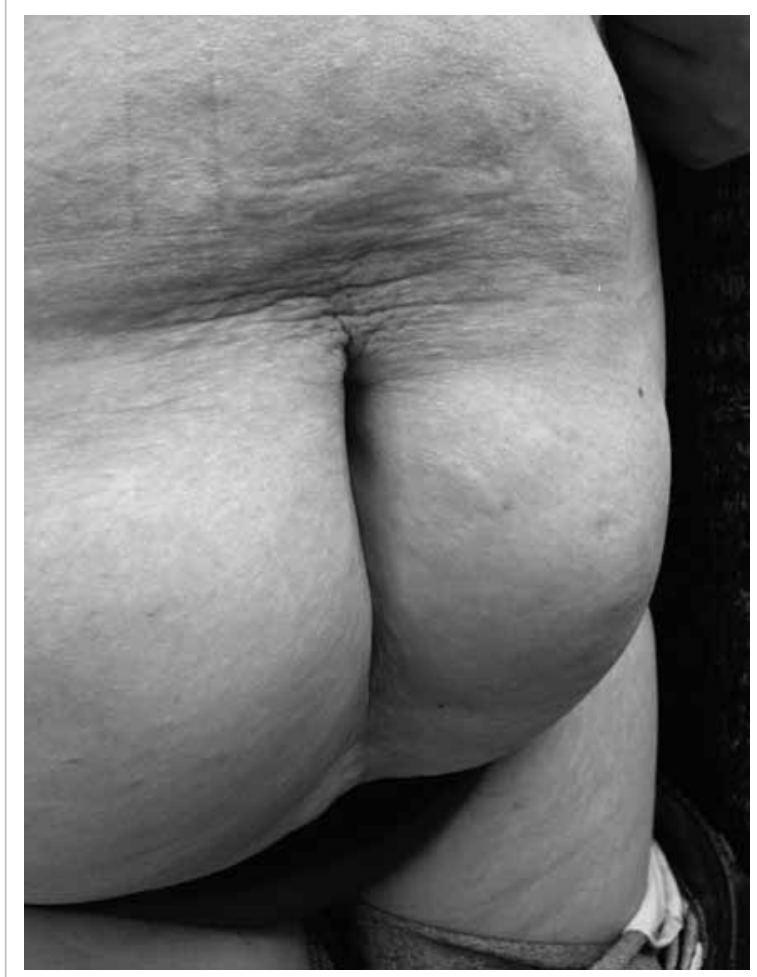

FIG I. Clinical picture of the pendular lipohypertrophy over the anterior lower part of the abdomen in a diabetic patient with long-term insulin

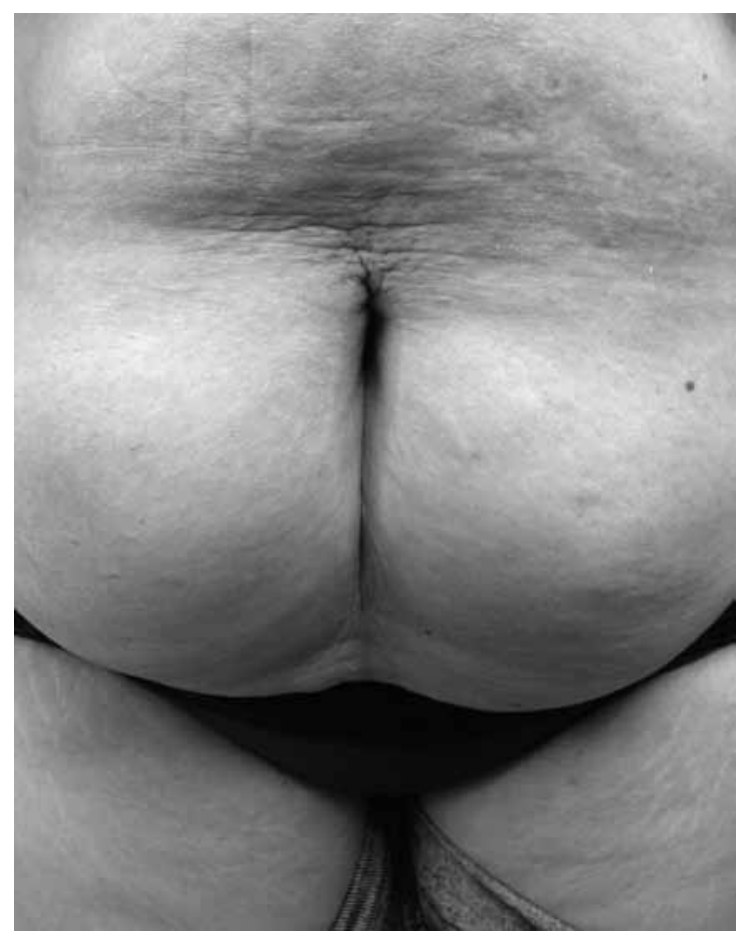

FIG 2. A 'cleft sign' in the midline flanked by the severe lipohypertrophy at the injection sites 
made during patient follow-up, especially in patients with unexplained fluctuations in glucose level. The glycated haemoglobin level of this woman was $6.8 \%$. Her insulin requirement was 81 units per day. She had occasional hypoglycaemic episodes at midnight.

The pathogenesis of lipohypertrophy remains unknown, but is most probably related to the local anabolic effect of insulin. Daily injection of a glucagonlike protein-1 agonist has not been reported to cause lipohypertrophy, ${ }^{3}$ making it unlikely for mechanical trauma to be an important factor. Immunogenicity also contributes little, since change to highly purified human insulin has not decreased the incidence.

Avoidance of risk factors such as repeated injection into the same sites, re-use of needles, and multiple injections is important. Regression of lipohypertrophy usually occurs over time if further injections into the affected areas are avoided. Nonetheless, caution should be exercised when switching to other sites because insulin requirement may be reduced. ${ }^{3}$ Liposuction may be considered when there is serious cosmetic concern. ${ }^{4}$

CM Ng ${ }^{*}$, FRCP (Edin), FHKAM (Medicine)

OL Chui, MNurs

SC Tiu, FRCP (Lond), FHKAM (Medicine)

Department of Medicine, Queen Elizabeth Hospital, Jordan, Hong Kong

* Corresponding author: ngcm2@ha.org.hk

\section{References}

1. Blanco M, Hernández MT, Strauss KW, Amaya M. Prevalence and risk factors of lipohypertrophy in insulin-injecting patients with diabetes. Diabetes Metab 2013;39:445-53.

2. Young RJ, Hannan WJ, Frier BM, Steel JM, Duncan LJ. Diabetic lipohypertrophy delays insulin absorption. Diabetes Care 1984;7:479-80.

3. Pledger J, Hicks D, Kirkland F, et al. Importance of injection technique in diabetes. J Diabetes Nurs 2012;16:160-5.

4. Hardy KJ, Gill GV, Bryson JR. Severe insulin-induced lipohypertrophy successfully treated by liposuction. Diabetes Care 1993;16:929-30. 